\title{
OTONOMIIK DISSFONKSİYON
}

\author{
AUTONOMIC DYSFUNCTION
}

Serdar KESKEN
Yaşar ZORLU

\section{SUMMARY}

The term autonomic dysfunction (or autonomic failure) includes disorders of the autonomic nervous system that appear in the progression of some diseases and causing in various conditions.

Diagnosis has been delayed frequently because of the unknown mechanisms between clinical symptoms and pathophysiology.

In this paper the causes and procedures of autonomic nervous system dysfunction are reviewed.

(Key Words: Autonomic failure)

\section{ÖZET}

Otonomik disfonksiyon ya da otonomik yetmezlik kavramları, otonom sinir sisteminin çeşitli nedenlerle ve bazı hastalıkların seyrinde ortaya çıkan bozukluklarını tanımlar.

Semptomların çeşitliliği ve otonom sinir sistemi fizyopatolojisinin yeterince bilinmemesi siklikla tanida gecikmelere neden olur.

Bu yazıda otonomik disfonksiyon nedenleri ve tanı yöntemleri kısaca gözden geçirilmiştir.

(Anahtar Sözcükler: Otonom yetmezlik)

\footnotetext{
Nöroloji Kliniği

SSK Tepecik Hastanesi 35120-iZMiR

(Uz. Dr. Y Zorlu Şef Yard., Uz. Dr. S Kesken)
} 
Otonom sinir sistemi, sinir sisteminin önemli bir bölümüdür. Fonksiyonlarının önemi ve çeşitliliği nedeniyle sinir sistemi hastalıkları ile ugraşan Nörologları ve viseral bozukluklar nedeniyle de İç Hastalıkları Hekimlerini yakından ilgilendirir. Bu sistem, kompleks santral bağlantıları ve yaygın periferik dağılımı ile canlılı̆̆ımızın devamı-nı, sürekli değişen iç ve dış çevre koşullarına uyumumuzu sağlar $(1,2)$. Otonomik disfonksiyon ya da otonomik yetmezlik, otonomi sinir sisteminin çesitli nedenlerle ve bazı hastalikların seyrinde ortaya çıkan bozukluklarını tanımlar (Tablo:2) (3). Ancak pratikte otonom sinir sistemi üzerinde yeterince durulmadığı da bir gerçektir.

Sinir sisteminin bu önemli bölümü ile ilgili çalışmalar, Galen'e (M.S.2. yüzyıl) kadar uzanır. Günümüze dek bir çok araştırıcı; sistemin anatomisi, işleyişi ve işlevleri konusunda çok sayıda bilgiler sunmuşlardır (4). Auerbach ve Meissner (1864), Henle (1868), Bouchardat ve Pavy (1885), Gaskell (1886); otonomik fonksiyon bozukluklarına değinen ilk araştırmacılardır. Daha sonra Bradburg ve Eggleston (1925) idiopatik (postural) hipotansiyon tanımını ortaya atmışlardır (5). Rundles (1945) ve Lamb (1960); savaş pilotlarında ve uzay yolculukları sırasında astronotlarda, yerçekimsizlik nedeniyle gelişen kardiovasküler problemlere yönelik araștırmaları sırasında otonomik yetmezlik kavraminı sıkça kullanmışlardır $(4,5,6)$.

Bu inceleme yazısında, otonomik disfonksiyon'a ilişkin bilgiler, klinik ve nedenlere yönelik sinıflama ile test yöntemleri, kısaca tedavi yaklaşımları gözden geçirilecektir.

\section{KLINIIK VE SINIFLAMA}

Otonomik disfonksiyonun neden olduğu çeşitli yakınmalar, hastaların sürekli hekimlere başvurmasına ve hekim değiştirmesine neden olmaktadır. Çoğunlukla kardiovasküler otonomik disfonksiyonun sonucu olarak ortaya çıan postural (ortostatik) hipotansiyona bağlı; başdönmesi, göz kararması, den- gesizlik, düşme atakları, çarpıntı gibi yakınmalarla karşılaşılmaktadır. Insanda alt ekstremitelerde, periferde ve visserlerde kanın toplanmasını engelleyen etkin bir kardiovasküler refleks kontrol mevcuttur. Bu mekanizma her türlüi iç ve dış etkiye karşı hızla yanit verecek durumda olmalidir. Otonomik yetmezlik başgösterdiğinde, bu denge bozulur ve intravasküler volüm hızlı yer değişimini sağlayamaz $(7,8)$. Postural, kardiovasküler yakınmalar sık olmakla birlikte, gastrointestinal, genitoüriner, seksüel ve sekretuar işlevler de bozulabilmektedir. Sistemlere ilişkin yakınmalar (Tablo:1) de sunulmuştur.

\section{TABLO 1: Otonomik Disfonksiyon Yakınma ve Bulguları}

\section{Postural:}

Başdönmesi, göz kararması, dengesizlik, düşme atakları, çarpıntı, uçlarda soğukluk.

2. Sekretuar:

Diş sekresyon, salivasyon ve terleme bozuklukları; ağız kuruluğu, hipersalivasyon, göz kuruması, terleme artışı veya azalması, sebore.

3. Gastrointestinal:

Yutma güçlüğü, aerofaji, karında gerginlik hissi, hazımsızlık, konstipasyon, kronik diyare ve gece diyareleri.

4. Ürogenital:

Miksiyon güçlüğü, idrar kaçırma, empotans (ereksiyon ve/veya ejakülasyon bozukluğu).

Diabetes mellitus, kronik azotemi, kronik alkolizm, toksik ve nutrisyonel olaylarla, Parkinson Hastalığı gibi primer hastalık olguları; ilerleyici bir süreç içinde otonomik isslevleri bozarak bu gibi yakınmalara neden olurlar $(9,10,11)$.

Otonomik disfonksiyonun eşlik ettiği primer hastalık ve sendromlar geniş bir yelpaze oluştururlar. Bu konuyla ilgili otoritelerce birçok sınıflamalar ortaya atılmıştır $(3,12)$. Ancak günümüzde çoğunlukla Mc Leod (1987)'ın periferik ve santral etkilenme temeline dayanan siniflaması kullanilmaktadır (Tablo:2) (3). 
TABLO 2: Otonomik Disionksiyonun Sınıflaması (Mc Leod, 1987)

1. SANTRAL

A. Pragressif otonomik yetmezlik

a. Idiopatik postural hipotansiyon

b. Parkinsonien progressif otonomik yetmezlik

c. Shy-Drager Sendromunda progressif otonomik yetmezlik

B. Parkinson Hastalığı

C. Medulla spinalis lezyonları

D. Wernicke ansefalopatisi

E. Diğerleri: Serebrovasküler hastalıklar, multipl skleroz, tabes dorsalis, beyin sap। tümörleri.

\section{PERIFERIK}

A. Saf otonomik nöropati:

(Akut ve subakut)

Pandisotonomi, kolinerjik disotonomi, botulismus.

B. Periferik nöropati ile birlikte otonomik nöropati. (Mikst nöropati)

a. Çarpıcı otonomik disfonksiyon: Diabetes mellitus, amiloidoz, akut enflamatuar nöropati, akut intermittan porfiri, RileyDay Sendromu.

b.llımlı otonomik disfonksiyon: Alkolik nöropati, toksik nöropati, vit. B-12 yetmezliği, sistemik lupus eritematosus mikst bağ dokusu hastalığı, kronik enflamatuar nöropati, Fabry Hastalığı.

\section{TABLO 3: Otonomik Disfonksiyon Testleri}

1. Kardiovasküler

2. Farmakolojik

3. Terleme ve Isı regulasyonu

4. Dış salgı bezleri

5. Gastrointestinal

6. Ürogenital

7. Elektrofizyolojik (deri potansiyelleri)

\section{ANATOMOPATOLOJI}

Otonom yetmezlikte, otonom sinir sisteminin santral ve periferik bölümlerinde histopatolojik, dejeneratif, nörokimyasal (nörotransmittör) değişiklikler olduğu bilinmektedir $(2,13,14,15,16)$.
Santrol otonomik kontrolde, hipotalamus önemli bir role sahiptir. Afferent ve efferent bağlantıları ile; kranial sinir çekirdekleri, limbik sistem ve diğer bazal çekirdeklerle ilişkilidir. Ayrıca korteksde parasempatik ve sempatik işlevlerde rol alan özel alanların varlığı da bilinmektedir $(2,12,17,18)$. Santral nedenli otonomik disfonksiyon, klinikte sıklıkla Parkinson Hastalığında gözlenir (Tablo:2) (6). Parkinson Hastalığında ve Shy-Drager Sendromunda (Serebral multisistem atrofi) post-mortem incelemelerde; hipotalamusta, kranial sinir çekirdeklerinde ve bazal gaglionlarda nöronal kayıplara, hücrelerde hyalinize Levy cisimciklerine rastlanir $(3,6,19,20)$.

Periferik otonomik sistem, fonksiyonel ve anatomik olarak; parasempatik ve sempatik bölümlerden oluşmaktadır. Parasempatikler; $3,7,9$ ve 10 . kranial sinirlerin otonomik lifleri ile medulla spinalisin 2,3 ve 4 . lomber segmentlerinden çlkan periferik sinirlerin otonomik liflerinden oluşmaktadır. Sempatikler ise; medulla spinalisin servikal 8 ile lomber 3 arasindaki segmentlerinden çıan periferik sinirlerin otonomik liflerinden oluşmaktadir. Her iki sistemin, çeşitli effektör organlarda çok çeşitli ve birbirine zıt işlevleri vardır $(2,3,21)$. Sempatik sistem, organizmanın aktivasyonu sırasında oluşan iç ve diş koşullarla uğraş verirken enerji sarfeder ve yorulur. Parasempatikler bu sirada devreye girer ve organizmanin dinlenmesini enerji toplamasını sağlarlar. Parasempatik ve sempatik lifler çişitli klinik durumlardan etkilenirler (Tablo: 2) $(13,15)$. Diabetes mellitusta, toksik ve nutrisyonel nöropatilerde, amiloidoz ve kronik alkolizme bağlı nöropatilerin seyrinde hastalık süresi ile artan oranlarda; myelinli liflerde aksonal yikım, vakuolizasyon ve sekestrasyonlara rastlanur $(15,22)$. Prevertebral sempatik ganglionlarda dendritlerde şişme, mezenterik sinirler ve visseral pleksuslarda preganglionik parasempatik liflerde dejenerasyon bulguları sık görülmektedir $(10,13$, 15). 


\section{OTONOM FONKSIYYN TESTLERII}

Otonom sistemi değerlendirmede kullanılan çok sayıda yöntem bulunmaktadır. Bunların bir bölümü kullanım ve uygulama güiçlüikleri nedeniyle terk edilmiştir. Halen uygulama alanı bulan otonomik disfonksiyon tanı yöntemleri güvenilir sonuç vermektedir. Ancak ilgili kliniklerin birlikte çalışmasını da gerektirecektir. Seçilmiş, güvenli test yöntemleri dört grubta toplanabilir $(1,12,28)$.

\section{Kardiovaskiiler testler}

- İstirahat nabzı: Hastanın 15 dakika yatak istirahati sonucu EKG trasesinde sayılan kardiak vuru sayısı dakikada 100 ve üstünde ise parasempatik tutuluşu destekler.

- Derin solunum testi: 1 dakikada 6 derin solunum sonrası hastanın EKG trasesinde en uzun ve en kısa $R-R$ aralığı saptanır. Iki aralık arasında kalb atım hızı 10 vuru/dak. dan küçük ise parasempatik tutuluşu destekler.

- Ayakta durmaya kalb hizı yanitı: Istirahat halinde iken ayağa kaldırılan hastada EKG trasesinde 30 ile 15 . vurunun R-R araliklarının milisaniye $(\mathrm{mS})$ cinsinden birbirine oranı 1,04 ve bundan küçükse sempatik tutuluşu destekler.

- Valsalva oranı: Hastaya basitçe Valsalva manevrası yaptırılırken EKG trasesinde 15 saniye içinde geçen en uzun ve en kısa R-R aralıklarının milisaniye ( $\mathrm{mS}$ ) cinsinden birbirine oranı 1,10 ve bundan küçükse parasempatik tutuluşu destekler.

- Postural hipotansiyon aranması: Tam istirahatte ve ayağa kaldırıldıktan 1 dakika sonra ölçülen sistolik arteriel basınç farkı 30 $\mathrm{mmHg}$ ve daha fazla düșme eğliminde ise sempatik tutuluşu destekler.

2. Pupil reaksiyon testleri

- Pilokarpin testi: \%0,16 lik pilokarpin hidroklorid solusyonu, normal oda ışığında pupil çapları ölçülüp kaydedildikten sonra her iki göze damlatılır. 10, 30, 45 ve 60 . dakikalarda pupil çapları tekrar ölçülür. Normalde beklenen myozis gelişmezse pa- rasempatik tutuluşu destekler $(12,28)$.

- Fenilefrin testi: \% 0,1 lik fenilefrin hidroklorid solusyonu, her iki göze pilokarpin testinde olduğu gibi uygulanır. Midriazis gelişmesinde defekt sempatik tutuluşu destekler $(23,24,28)$.

3. Lakrimal sekresyon testi (Schirmer Testi): Her iki göz dıs konjunktiva kesesine özel test kağıtları yerleștirilir. 5 dakikalık basal gözyaşı salgısı kağıt üzerinde $5 \mathrm{~mm}$ den az ilerledi ise paresempatik defekt sözkonusudur $(23,25)$.

4. Deri potansiyel kayıtllaması: Avuç içi ve el sırtı ile ayak tabanı ve ayak sırtına uygulanan yüzeyel elektrodlar EMG cihazına bağlanır. Uyarıcı bipolar eletrod ile üst akstremitede median, alt ekstremitede peroneal sinirler $0,2 \mathrm{mS}$ süreli $40 \mathrm{~mA}$ gücünde elektrik şok ile uyarılarak deri potansiyelleri kaydedilir. Kaydedilen potansiyelin latans (S) ve amplitüd ( $\mathrm{mV}$ ) değerleri, normal değerlerle karşılaştırıldığında latans uzaması, amplitüd düşmesi sempatik tutulus lehine değerlendirilir $(26,27)$.

Literatürde yer alan diğer test yöntemleri de grub bașlikları altında tablo halinde toparlanılmaya çalıșılmıştır (Tablo: 3) (1, 12, 28).

\section{YORUM}

Otonom sinir sisteminin anatomisi, fizyolojisi ve farmakolojisi yakın zamana kadar iyi bilinmemekteydi. Tip teknolojisi ve moleküler nörobiyolojide ortaya konulan yenilikler bu konuda bir çok billinmeyeni açıklığa kavușturmuștur. Araștırmalar çoğunlukla hayvanlar üzerinde yapılmakta, farmakolojik testler ve elektriksel stimülasyon yöntemleri kullanılmaktadır (1, 13, 14). Santral otonom yapilarla, santral kimyasal defektlerin ilișkisi derinlemesine araștırılmaktadır. Özellikle inferior oliver nukleusta, dentat nukleusta ve gama amino butirik asit (GABA) içeren nukleuslarda nöro-ileticilerde azalma olduğu bilinmektedir. Bu gibi yapılarda hücre kayıpları ile paralel oranda glutamat ve as- 
partat düzeyinde de azalma olduğu bildirilmektedir. Ayrıca bazı önemli iletim bileşkelerinde de norepinefrin düzeylerinde kayıplar gözlenmektedir $(15,17,21)$.

Son araştırmalarda; kardiovasküler ve solunumsal işlevlerin pontin parabrakial nukleusta (PPN) ve nukleus traktus solitariusta (NTS) oluştuğu ve buralardan da rostroventrolateral medullaya (RVLM) projekte olduğu gösterilmiştir. Bu iki yapının baroreseptor ve kemoreseptor reflekslerin merkezi olduğu bilinmektedir $(8,29,30)$.

Bir çok sistemik ve nörolojik hastalığın seyrine eşlik eden otonomik disfonksiyon, santral nedenle en çok Parkinson Hastalığında karşımıza çıkmaktadır. Bunun yanısıra daha çok postural hipotansiyonla seyreden ilerleyici idiopatik otonomik yetmezlik tablosunu da gözardı etmemek gerekmektedir $(19,20,31,32,33)$.

Periferik nedenli otonomik disfonksiyon ise çoğunlukla Diabetes Mellitusa eşlik eder $(10,11,31)$. Alkolik ve toksik nöropatiler, amiloidoz, akut ve kronik enflamatuar proçesler, bazı bağ dokusu hastalıkları ve sistemik lupus eritematosus ile porfiride de periferik etkilenme sonucunda otonomik disfonksiyon ortaya çıabilmektedir (Tablo: 2).

Klinisyene, primer hastalık tablosu ile ilgisizmiş gibi görünen bazı yakınmaların erken çözümünde, seçilmiş test yöntemlerinin uygulanması soruna yardimci olacaktır, kanaatindeyiz. Illgili kliniklerin yakın işbirliği ile söz konusu testler kolaylikla uygulanarak, faydalı sonuçlar elde edilebilir.

\section{TEDAVI YAKLAŞIMLARI}

Ortostatik hipotansiyonda fludrokortizon'un günde $0,1 \mathrm{mg}$ ile başlayan ve yavaş, yavaş artırılan dozlarının faydalı olduğu ifade edilmektedir. Efedrin ve fenilefrin hidroklorid gibi sempatomimetik drogların da kullanıldığı, yanısıra dihidroergotamin'in de venöz damar yatağında vazokonstriktör etkili olduğu bildirilmektedir. Monoaminooksidaz inhibitörleri ile tiramin'in kombine kullanılmasının özellikle postural hi- potansiyona etkili olduğu saptanmıştır.

Santral iletimde sempatik aktiviteyi bloke eden klonidin, periferik alfa-2 agonist etkisi ile primer venokonstriksiyona yol açarak yine bazı hastalarda yararlı olmaktadır $(12,28,33)$ :

Prostaglandin inhibitörlerinden indomatazin; fludrokortizon ile kombine halde kullanilmaktadır. Pnopranolol, metaklopramid, kafein, pindolol, prenalterol gibi bazı droglardan da çeşitli etkileri nedeniyle faydalanıldığı bildirilmektedir.

Domperidon ise vagal ve splanknik parasempatik bloker etkisinden yararlanılarak kusma, gastrik regürjitasyon ve özofagus atonilerinde kullanılmaktadır (7).

Tedavide yararlı olduğu söylenen ilaçların çoğu ülkemizde çeşitli isimlerle piyasada bulunmaktadir. Klinisyen, asıl hastalık olgusuna yönelik tedavi yanında; bu ilaçların da faydalı etkilerinden yararlanarak iyi sonuç alabilir.

\section{KAYNAKLAR}

1. Appenzeller $O$. The Autonomic Nervous System. 3- Amsterdam, Elsevier: 1982; 524

2.Clark GR. (Çev. Zileli T.) Manter and gatz's essentials of clinical neuroanatomy and neurophysiology. Ankara. Hacettepe Üniv Yay. 1979; 123-37.

3. Mc. Leod JG, Tuck RR. Disorders of the autonomic nerjous system, Part 1, Pathophysiology and clinical features. Ann Neurol. 1987; 21: 419-30.

4. White JC, Smithwick RH. The Autonomic Nervous System. New York. 1948; 469.

5. Bradbury S, Eggleston C. Postural hypotension: a report of three cases. Am J Heart. 1925; 1: 73-86.

6. Qrskov L. Autonomic function in Parkinsonian patients relates to duration of disease. Neurology. 1987; 37: 1173-8.

7. Destee A. Orthostatic hypotension due to diabetic autonomic neuropathy? Treatment with domperidone. Arch Neurol. 1987; 44: 11-2.

8. Guilleminault C, van den Hoed J, Mitler MM. Diabetic autonomic dysfunction, blood pressure and sleep. Ann Neurol. 1985; 18: 670-5.

9. Caird FI, Andrews GD, Kennedy RD. Effect of posture on blood pressure in the elderly. Br Heart $J$. 1973; 35: 527-30. 
10. Jeyarajaln R. Autononic function tests in noninsulin dependent (Type 2) diabetic patients and apparently henlthy zolunteers. Chron $j$ Disease. 1986; 39: $479-84$

11. Krönert K. Reduced intraindividual variability of repeated cardiozascular reflex tests: an additional marker of autonomic neuropathy in insulin dependent (Type 1) diabetes mellitus. Acta Diabetol. 1986; 23: 279-89.

12. Bannister R. A Textbook of Clinical Disorders of the Autonomic Nervous System. Oxford. Oxford University Press. 1983: 666.

13. Couen T, Thrasivoulou Cb Cerebrozascular nerces in old rats show reduced accumulation of 5 hydroxytryptamine an lose of nerve fibres. Brain Res. 1990; 513: 237-43.

14. Exing D). Clinical aspects of primary and secondary autononic failure. Neurol Neurosurg. 1991; 4: 539-44

15. Yaghaski S, Sima FAA. Diabetic neuropathy in $B B$ rat. Ultrastructural and morphometric changes in parasempathetic nerres. Diabetes. 1986; 35: 73343.

16. Yasui Y, Cechetto DF, Saper CB. Evidence for a cholinergic projection from the pedunculopontine tegmental mucleus to the rostral ventrolateral medulla in the rat. Brain Res. 1990; 517: 19-25.

17. Polinsky JR. Pharmacology of the autonomic nervous systen in man. Neurol Neurosurg. 1991; 4: 533-8.

18. Talman WT. Cardiozascular regulation and lesions of the central nerrous system. Ann Neurol. 1985; 18: 1-12.

19. Appenzeller O, Goss JE. Autonomic deficits in Parkinson's Syndrone.. Arch Neurol. 1974; 24: 50-7

20. Goetz CG, Lutge W, Tanner MC. Autonomic dysfunction in Parkinson' disense. Neurology. 1986; 36: $73-5$.

21. Senard JM. Decreased high affinity state in platelet $\quad \alpha-2$ adrenoceptors from diabetic patients with orthostatic hypotension. I Clin Endocrinol Metab. 1990; 71: 311-7.
22. Ryder R, Kennedy RI, Neurick Pg, Wilson RM, Ward JD, Hardisty CA. Autononic denerzation may be prerequisite of diabetic neuropathic foot $\mathrm{ul}$ ceration. Diabetic Med. 1990; 7: 726-30.

23. Kuntral K, Selçuki D, Çolakoğlu Z, Yaycıŏ̆lu S. Saf pandizotonomi. Nörolojik Bilimler Dergisi. $1990 ; 7: 48-50$.

24. Lowenstein O. Miosis in Arglyll-Robertson Syndrome and related pupillary disorders. Arch Ophtalmol. $1986 ; 55: 356-70$.

25. Hanson J, Fikentscher R, Roseburg B. Schirner's test of lacrimation, its clinical importance. Arch Otolaryngol. 1985; 101: 293-5.

26. Ertekin C, Ertekin N, Mutlu S, Almis S, Akçam A, Skin potentials (SP) recorded from the extrenities and genital regions in nomnl and impotent subjects. Acta Neurol Scand. 1987; 76: 28-36.

27. Knezeuric W, Bajda S. Peripheral autonomic surface potential. I Neurol Neurosurg Psychiatry. $1985 ; 25: 126-33$

28. MC Leod JG, Tuck RR. Disorders of the aufononic nerrous system, Part 2, Investigation and treatment. Ann Neurol. 1987; 22:519-29.

29. Kronenberg MW, Forntan Mb, Onrot J, Robettson $D$. Enhanced left ventricular contractility in autonomic failure: Assesment using pressure rolume relations. I An Coll Cardiol. 1990; 40: 1334-42.

30. Spyer KM. Physiology of the autononic nerrous system: CNS control of the cardiowascular system. Neurol Neurosurg. 1991; 4: 528-32.

31. Bennet T, Farquar IK, Hosking DJ, Hanipton IR. Assesment of methods for estimating autonomic nervous control of the heart in patient with diabetes mellitus. Diabetes. 1983; 27: 1167-74.

32. Ewing DJ. The clinical features of non-diabetic autonomic failure. Scot Med J. 1990; 35: 35-8.

33. Ziegler MG. Postural hypotension. Prev Med. 1980; 31: $239-45$. 